\title{
Single Digital Predistortion Technique for Phased Array Linearization
}

\author{
Sara Hesami*, Sina Rezaei Aghdam ${ }^{\dagger}$, Christian Fager $^{\ddagger}$, Thomas Eriksson ${ }^{\dagger}$, Ronan Farrell*, John Dooley* \\ Email: \{sara.hesami,ronan.farrell,john.dooley\}@mu.ie \{sinar,christian.fager,thomase\}@ chalmers.se \\ *Department of Electronic Engineering, Maynooth University, Ireland. \\ ${ }^{\dagger}$ Department of Electrical Engineering, Chalmers University of Technology, Gothenburg, Sweden. \\ $\ddagger$ Department of Microtechnology and Nanoscience, Chalmers University of Technology, Gothenburg, Sweden.
}

\begin{abstract}
In this paper, we present a novel and effective linearization technique for nonlinear phased array antennas. For large phased arrays, linearization of the array using a single digital predistortion (DPD) is inevitable since one digital path is upconverted and feeds several RF transmission paths, each of which is connected to a power amplifier (PA) and an antenna element. However, a critical issue is that the PA characteristics can vary considerably within an array. Thus, linearizing individual PAs with one DPD is rather challenging. We formulate and solve an optimization problem that corresponds to jointly minimizing the maximum residuals between the input to the array and the output of individual PAs. We demonstrate that the proposed technique outperforms state-of-the-art linearization solutions while retaining the linear gain of the array.

Index Terms - digital predistortion, linearization, phased array, single DPD, MIMO.
\end{abstract}

\section{INTRODUCTION}

The requirement for energy and spectral efficiency for fifth generation $(5 \mathrm{G})$ wireless networks has triggered the advancement of several technologies, one of which is large scale phased array for beamforming. Further, the use of hybrid arrays, with multiple sub-phased arrays for multiuser capacity, is therefore anticipated to be the key enabler for 5G [1]. Phased array facilitates the adaptation of sub-millimeter and millimeter-wave (mm-wave) spectrum. A large number of antennas improves the throughput by focusing the radiated signal into a small region. Thus it can overcome high path loss in mm-wave [1] as well as reducing the power per power amplifier (PA) for both sub-millimeter wave and $\mathrm{mm}$-wave. Another advantage of the large phased array is the use of low-cost components in multiple radio frequency (RF) chains, each of which is connected to an antenna element.

To improve the energy efficiency in small and low cost power amplifiers (PAs), it has been suggested to drive the PAs close to saturation where PAs have nonlinear behavior. Hardware impairments such as PA nonlinearity can fundamentally limit the performance of the base station (BS). A study by [2] have indicated that $4 \mathrm{~dB}$ back-off was required to achieve the error vector magnitude (EVM) lower than $-29 \mathrm{dBc}$. However, applying back-off to overcome the nonlinearity of the PAs requires using higher power PAs to give a similar performance while sacrificing the energy efficiency of the transmitter. A better approach to overcome the PA efficiency vs. linearity trade-off is to drive the PA close to saturation while linearizing it.

Digital predistortion (DPD) is a widely accepted solution for PA linearization, and it relies on distorting the input of the PA such that the distortions due to the nonlinear effects of the PA are compensated [3]. A typical implementation of the DPD technique is done in digital baseband and requires as many feedback paths and predistorders as the number of power amplifiers. However, for phased array systems where one digital baseband branch is connected to several analog transmission paths in RF, only a single DPD can be implemented per sub-array. This single DPD is expected to linearize several PAs, each of which can have different characteristics. Different strategies can be applied to optimise the single DPD performance. In [4], the authors propose a single DPD technique to linearize multiple PAs in a phased array. Their proposed solution relies on minimizing the sum of the least squared errors of the different branches. A beam-oriented single DPD is introduced in [5] where a single DPD is employed to linearize the main beam signal. The proposed DPD is based on minimization of the least square error of the superposition of the PA outputs after the effect of analog beamformers are removed. An array DPD was introduced by [6], where the combined error in the far-field is minimized. The combined error is estimated by taking the feedbacks from all PAs and calculating the combined response of the array in the far-field and comparing it with the input signal to the PAs. The work in [7] is using the same approach by cancelling the beamforming weights before combining via an anti-beamforming module. Further, the effect of phase and amplitude mismatch of feedback signals on the DPD learning algorithm has been investigated. In [8], a single DPD is trained to linearize the sub-array by modeling the main beam signal and comparing it with the input signal and observe the signal linearity in the far-field.

In this paper, we perform a comparative study among different single DPD solutions for phased array systems with combined feedback response and with individual PA feedback responses. By proposing a novel single DPD method, we formulate a convex optimization problem which relies upon on joint minimization of the cost functions from all transmission branches. We demonstrate that the proposed solution outperforms the ones in [4]-[8]. This improved performance 
is especially notable when the PAs in the different branches possess considerably different characteristics. The DPD learning utilizes a closed-loop estimation technique which is not sensitive to the PA output noisy measurements.

The remainder of the paper is organized as follows. In Section II, a system model for a single DPD for a sub-array is represented. In Section III, two previous models for single DPD are revisited, and a new DPD method is presented. In Section IV, the simulation results are given for different single DPD methods. Finally, a conclusion is given in Section V.

\section{System Model For Single DPD in A BEAMFORMING SUB-ARRAY}

A block diagram of analog beamforming phased array with a single DPD is depicted in Fig.1. The PA outputs can be obtained using a shared feedback path connected to each transmission path by a switch. To have access to all PA outputs, time sharing can be used. The complex baseband equivalent of the PA output can be written as a polynomial model [9]. The nonlinear memory effects are typically weaker than the nonlinearities without memory [10], and we omit the memory effect here, but it is easy to generalize to memory if desired. The baseband equivalent of PA output in the $i$ th transmission path when no DPD is applied can be expressed as

$$
y_{i}(n)=v_{i} \boldsymbol{\psi}_{x_{i}}^{T}(n) \mathbf{h}_{i}, i=1, \ldots, N_{T},
$$

where $N_{T}$ is the number of PAs, $\boldsymbol{\psi}_{x_{i}}(n)=$ $\left[x(n), x(n)|x(n)|^{2}, \ldots, x(n)|x(n)|^{p_{i}-1}\right]^{T} \quad$ is the basis waveform and $v_{i}$ represents the analog beamforming phase shifters in the $i$ th transmitter path. The vector $\mathbf{h}_{i}$ denotes the $i$ th PA model impulse response and $p_{i}$ is the order of nonlinearity in the $i$ th PA. In a multi-transmitter system, the individual PAs have different non-linearity characteristics that can be presented by different model coefficients. However, the combined response of the array still can be modeled with a single polynomial. Given this fact, the single DPD for a sub-array can be modeled in the form of a polynomial as

$$
x_{\mathrm{DPD}}(n)=\boldsymbol{\psi}_{x}^{T}(n) \boldsymbol{\omega},
$$

where $\boldsymbol{\omega} \in \mathbb{C}^{p \times 1}$ is the vector of the DPD coefficients for the basis waveforms $\psi_{x}(n)=$ $\left[x(n), x(n)|x(n)|^{2}, \ldots, x(n)|x(n)|^{p-1}\right]^{T}$. The model is linear in $\boldsymbol{\omega}$, and thus the model can be fitted with the least squares approach. Utilizing a closed-loop learning DPD, to obtain the optimum DPD coefficients, the difference between the desired output signal and the actual PA output is obtained as

$$
e_{i}(n)=\frac{1}{G_{i}} \tilde{y}_{i}(n)-x(n),
$$

for each path where $G_{i}$ is the complex linear gain of the $i$ th PA. The linear gain of the PA can be known by using the least square approach between the PA input and output. The phase offsets in output feedback signals are introduced by analog phase shifters which can degrade the performance of the single DPD. Thus, the phase offsets must be taken into account prior

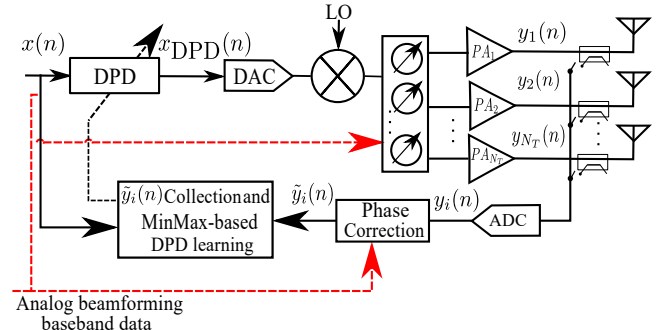

Fig. 1: Block diagram of a sub-array to access each PA units output with shared feedback.

to DPD learning either by canceling the effect of phase shifters in feedback path or modeling the equivalent far-field model of individual PA outputs. Modeling the output signals in the farfield can limit the performance of DPD to a specific direction while, counteracting the effect of phase shifters in the feedback signals, facilitates the adoption of trained DPD coefficients for different steering directions. Thus, we suggest a phase correction such that the signal $\tilde{y}_{i}(n)$ is obtained as

$$
\tilde{y}_{i}(n)=v_{i}^{*} y_{i}(n) .
$$

In the closed-loop learning, the estimation loop contains the DPD within itself. Thus, the estimated parameter corresponds to the residual error in the DPD coefficients [11]. For a block of time samples, the cost function to be minimized reads as

$$
\mathrm{J}_{\mathrm{CL}}=\left\|\boldsymbol{e}_{i}-\boldsymbol{\Psi}_{x}^{T} \Delta \boldsymbol{\omega}\right\|_{F}^{2},
$$

where $\Delta \boldsymbol{\omega} \in \mathbb{C}^{p \times 1}$ is the vector of the residual model coefficients, $p$ represents the order of nonlinearity and \|\|$_{F}$ represents the Frobenius norm. Through a few iterations the DPD coefficient can be updated. First, by utilizing the leastsquares, the estimation of coefficient residual is obtained as

$$
\Delta \boldsymbol{\omega}=\left(\boldsymbol{\Psi}_{x}\right)^{\dagger} \boldsymbol{e}_{i},
$$

where the matrix $\Psi_{x}$ contains the blocks of time samples of basis waveforms and $\left(\boldsymbol{\Psi}_{x}\right)^{\dagger}=\left(\mathbf{\Psi}_{x}^{H} \mathbf{\Psi}_{x}\right)^{-1} \mathbf{\Psi}_{x}^{H}$ is the pseudoinverse. Then, the coefficients are updated as follows

$$
\boldsymbol{\omega}^{i+1}=\boldsymbol{\omega}^{i}+\Delta \boldsymbol{\omega}
$$

where $\boldsymbol{\omega}^{0}=[1,0, \ldots, 0]$.

\section{BEAMFORMing DPD With MinMax}

Various studies have used different methods to estimate the single DPD coefficients. These can be classified in two categories, combined output of the array response [5]-[8] or Combined Feedback DPD, and the sum over the least square errors [4] or Sum of Errors DPD. First, we present these two methods in a closed-loop learning form. Then, we propose a novel DPD method, noted as MinMax DPD. Finally, we perform a comparison between these three DPD methods. It has been shown in [12] that the out-of-band radiations are not dominant in the directions other than the direction of UE. This is the primary motivation behind the Combined Feedback DPD as it considers the combined response of the array in far-field. Combined Feedback DPD can be implemented either via a 
combined feedback receiver signal [5], [7] or by modeling the combined far-field signal [6], [8]. In both techniques, the effect of analog phase shifters is removed before DPD learning. To minimize the array error with respect to far-field combined signal, a large block of error samples can be obtained by comparing the combined far-field signal at the position of UE and the input signal. In order to calculate far-field combined signal, the feedback signals are co-phased and combined. An alternative approach is to model the far-field response of the array. Combined Feedback DPD is based on the minimization as follow

$$
\underset{\Delta \boldsymbol{\omega} \in \mathbb{C}^{p \times 1}}{\arg \min }\left\|\left(\frac{1}{G_{A}} \sum_{i=1}^{N_{T}} \tilde{\boldsymbol{y}}_{i}-\boldsymbol{x}\right)-\boldsymbol{\Psi}_{x}^{T} \Delta \boldsymbol{\omega}\right\|_{F}^{2},
$$

where $G_{A}$ represents the array linear gain and the signals $\boldsymbol{x}=[x(n), x(n-1), \ldots, x(n-k)]^{T}$ and $\boldsymbol{y}_{i}=\left[y_{i}(n), y_{i}(n-\right.$ 1), .., $\left.y_{i}(n-k)\right]^{T}$.

Another approach, Sum of Errors DPD, is introduced in [4], minimizes the sum over the least square errors, i.e., the sum of cost functions in (5) is minimized as

$$
\underset{\Delta \boldsymbol{\omega} \in \mathbb{C}^{p \times 1}}{\arg \min } \sum_{i=1}^{N_{T}}\left\|\left(\frac{1}{G_{i}} \tilde{\boldsymbol{y}}_{i}-\boldsymbol{x}\right)-\boldsymbol{\Psi}_{x}^{T} \Delta \boldsymbol{\omega}\right\|_{F}^{2} .
$$

In this approach, the error signal for each PA output signal is computed individually and the sum of them is minimized to obtain the optimum solution for $\boldsymbol{\omega}$. For the sake of consistency we performed co-phasing of the PA output signals in the same way it has been implemented for Combined Feedback DPD. Exploiting triangle inequality, it can be inferred that the performance of (8) outperforms (9) in the desired direction [6]. However, in both techniques, the PA output with the most deviation from the reference signal $x(n)$, has the dominant contribution in the cost function minimization. Consequently, by linearizing the highly nonlinear PAs, the PAs with lower nonlinearity order heavily expand and degrade the combined performance of the array [6]. To address this issue, we propose a novel strategy by utilizing individual cost functions. The model coefficients must then be estimated to minimize all the cost functions up to an acceptable range. In order to minimize all the cost functions jointly, we formulate a convex optimization problem as

$$
\underset{\Delta \boldsymbol{\omega} \in \mathbb{C}^{p \times 1}}{\arg \min } \max _{i=1, \ldots, N_{T}}\left\|\left(\frac{1}{G_{i}} \tilde{\boldsymbol{y}}_{i}-\boldsymbol{x}\right)-\boldsymbol{\Psi}_{x}^{T} \Delta \boldsymbol{\omega}\right\|_{F}^{2},
$$

where the model coefficient residuals, $\Delta \omega$ is the variable to optimize. A careful reader must notice that this optimization problem does not imply minimization of one cost function related to the most nonlinear PA. Instead, (10) performs an optimization where the maximum joint error is minimized. Introducing an auxiliary variable $d$ representing the minimum
Euclidean distance, the optimization problem in (10) can be equivalently written as

$$
\begin{aligned}
& \underset{\Delta \boldsymbol{\omega} \in \mathbb{C}^{p \times 1}, d \in \mathbb{R}}{\operatorname{minimize}} d \\
& \text { subject to }\left\|\left(\frac{1}{G_{i}} \tilde{\boldsymbol{y}}_{i}-\boldsymbol{x}\right)-\boldsymbol{\Psi}_{x}^{T} \Delta \boldsymbol{\omega}\right\|_{F}^{2} \leq d, i=1, \ldots, N_{T} .
\end{aligned}
$$

It can be seen that in the above formulation the quadratic constraints that represent the minimum Frobenius norm squared, are convex with respect to $\boldsymbol{\omega}$. Also, the object $d$ is convex with respect to $\boldsymbol{\omega}$. Thus, the above standard optimization problem can be efficiently solved using standard techniques [13]. We used CVX to specify and solve the convex problem in (11) [14], [15].

\section{Simulation Results And Analysis}

As a proof of concept, we perform quantitative performance analysis. The system level simulation for a sub-array with four transmit antennas in a uniform linear array (ULA) with the spacing of half wavelength is implemented in Matlab. In all experiments, the PAs are modeled with a memoryless complex polynomial. First, the PA models for a 3rd and 5 th order polynomials are estimated. Then, we obtain the models for the PAs in the adjacent branches by adding a small perturbation to the 3rd and 5th order nonlinearity coefficients such that different PA behaviors are obtained while retaining the similar input-output nonlinear characteristics and linear gain for all PAs. Orthogonal frequency-division multiplexing (OFDM) signal with $5 \mathrm{MHz}$ bandwidth is used where clipping is applied to the input signal to obtain peak-to-average power ratio (PAPR) of $8.5 \mathrm{~dB}$ before linearization. To evaluate the performances, the adjacent channel leakage ratio (ACLR) is used to calculate the out-of-band radiation of the distortion in the adjacent channels due to the nonlinearity in the system. The ACLR for a signal $x(n)$ is formulated as

$$
A C P R=\max _{m=L, U}\left\{\frac{\sum_{f(a d j)_{m}}|X(f)|^{2}}{\sum_{f c h .}|X(f)|^{2}}\right\},
$$

where $X(f)$ denotes the power spectrum of $x(n)$ and $f(a d j)_{m}$ represents the frequencies in lower (L) and upper (U) adjacent channels. The $f c h$ represents the inband frequencies. The results for the DPD training of $N=12500$ samples with SNR of $40 \mathrm{~dB}$ are presented. The combined far-field response of array is studied when three different DPDs are implemented and trained for the sub-array when the steering angle is set to $30^{\circ}$. In Fig. 2a and Fig. 2b the power spectral densities (PSD) of the overall array are depicted for 3rd and 5th order nonlinearity with three different DPDs. Clearly, MinMax DPD outperforms Combined Feedback and Sum of Errors DPDs. To quantitatively study the performances, the ACLR results are presented in Table I. For 3rd order polynomial, the MinMax performs on average $2.82 \mathrm{~dB}$ better in ACLR measurements. However, as the polynomial order increases to 5, MinMAx DPD outperforms two other DPD by $11 \mathrm{~dB}$ on average. Comparing 3rd and 5th order nonlinear models, 
TABLE I: ACLR (dB) evaluation of three different DPD methods for different PA models.

\begin{tabular}{c|c|c}
\hline \hline DPD method & $\begin{array}{c}\text { 3rd Order } \\
\text { PA model }\end{array}$ & $\begin{array}{c}\text { 5th Order } \\
\text { PA model }\end{array}$ \\
\hline \hline Sum of Errors & -39.91 & -27.58 \\
\hline Combined Feedback & -41.65 & -33.56 \\
\hline MinMax & -43.65 & -41.66 \\
\hline
\end{tabular}

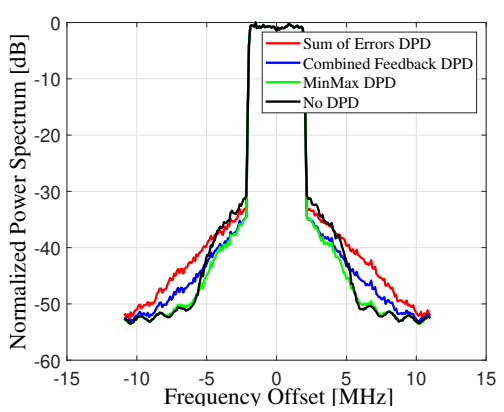

(a)

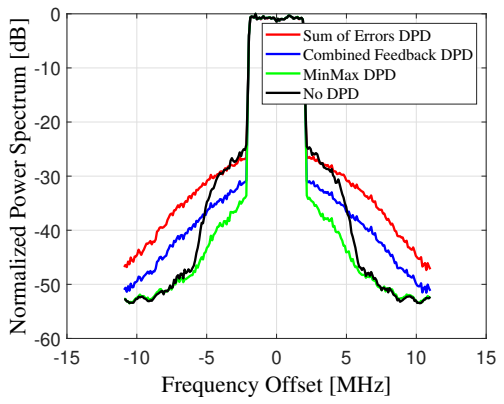

(b)

Fig. 2: Spectra of the combined array response in the far-field for different DPD methods with PA models of (a) 3rd (b) 5th order polynomial.

MinMax performance does not degrade more than $2 \mathrm{~dB}$ while the ACLR for Combined Feedback DPD worsens by $8 \mathrm{~dB}$ and Sum of Errors DPD by $12.33 \mathrm{~dB}$. The reason that the MinMax DPD performance does not degrade considerably by increasing the nonlinearity order of the PAs is the fact that MinMax DPD does not depend on the superposition of signals. Rather, the MinMax consider each error signal individually. However, in the Sum of Errors DPD, as the nonlinearity increases the superposition of error signals results in performance degradation. In Combined Feedback DPD, the combined far-field signal deviates further from the input signal as the nonlinearity of the PAs increases.

In Fig. 3, the AM/AM response of individual PAs and the combined effect of the three different DPD approaches are presented. It is evident that the combined effect of the MinMax results in considerably more linear combined behavior. In the simulations, it was observed that for the 3rd order PA model when no DPD is applied, the combined response of the array shows significant linearity. This is because the nonlinearities in different PAs can cancel each other out in the far-field. However, when no DPD is applied, the linear gain of the array

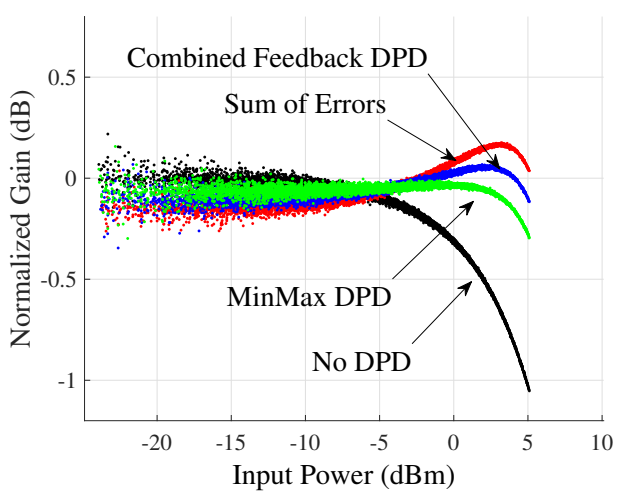

Fig. 3: AM/AM plots for combined array far-field responses without DPD and with three different DPD methods with 3rd order PA models.

degrades as it is shown in Fig. 3. By implementing the DPD, the combined response of the signal retains the linear gain thus improving the efficiency. In this sense, MinMax DPD achieves best linearization while sustaining the linear gain of the array.

\section{CONCLUSION}

In this paper, we proposed a new technique for training a single DPD for linearization of a phased array. The proposed DPD implements a joint minimization of cost functions corresponding to individual PAs which is an effective method to obtain a linear combined response of an array. Our numerical results demonstrated that the proposed DPD technique is highly effective when there is a significant deviation in PA behaviors. With simulations, we showed that as the nonlinearity order in PAs increases, the proposed DPD sustains its linearization while the other methods undergo significant performance degradation. Our numerical results demonstrated that by using the proposed DPD technique, for the 5th order PA models, the MinMax DPD outperforms the state-of-the-art solutions. We showed that in comparison to Combined Feedback DPD, the MinMax DPD has a better performance of $8.1 \mathrm{~dB}$ in the ACLR measurements and outperforms Sum of Errors DPD by $14 \mathrm{~dB}$. For the 3rd order PA models, the combined far-field signal without DPD shows linear performance where the nonlinearities cancel each out other but the linear gain of the array drops by combining the linear PAs with heavily nonlinear PAs. We showed that the MinMax DPD performs linearization while it retains the linear gain of the array. The effect of memory is not considered in this study. The memory effect is well studied in the literature, and by updating the polynomial model used in this study to a memory polynomial, our approach can be considered for wideband signals.

\section{ACKNOWLEDGMENT}

This publication has emanated from research conducted with the financial support of Science Foundation Ireland (SFI) and is co-funded under the European Regional Development Fund under Grant Number 13/RC/2077. 


\section{REFERENCES}

[1] E. G. Larsson, O. Edfors, F. Tufvesson, and T. L. Marzetta, "Massive MIMO for next generation wireless systems," IEEE Commun. Mag., vol. 52, no. 2, pp. 186-195, Feb 2014.

[2] K. Kibaroglu, M. Sayginer, and G. M. Rebeiz, "A quad-core $2832 \mathrm{GHz}$ transmit/receive $5 \mathrm{G}$ phased-array IC with flip-chip packaging in $\mathrm{SiGe}$ BiCMOS," in IEEE MTT-S Int. Microwave Symposium (IMS), Jun 2017, pp. 1892-1894.

[3] G. Lazzarin, S. Pupolin, and A. Sarti, "Nonlinearity compensation in digital radio systems," IEEE Trans. Commun., vol. 42, no. 234, pp. 988999, Feb 1994.

[4] S. Lee, M. Kim, Y. Sirl, E. Jeong, S. Hong, S. Kim, and Y. H. Lee, "Digital predistortion for power amplifiers in hybrid MIMO systems with antenna subarrays," in IEEE 81st Vehicular Technology Conference (VTC Spring), May 2015, pp. 1-5.

[5] W. Chen, G. Lv, X. Liu, and Z. Feng, "Energy-efficient doherty power amplifier MMIC and beamforming-oriented digital predistortion for 5G massive MIMO application," in 2017 IEEE Asia Pacific Microwave Conference (APMC), Nov 2017, pp. 391-394.

[6] N. Tervo, J. Aikio, T. Tuovinen, T. Rahkonen, and A. Parssinen, "Digital predistortion of amplitude varying phased array utilising over-the-air combining," in IEEE MTT-S Int. Microwave Symposium (IMS), Jun 2017, pp. $1165-1168$

[7] M. Abdelaziz, L. Anttila, A. Brihuega, F. Tufvesson, and M. Valkama, "Digital predistortion for hybrid MIMO transmitters," IEEE J. Sel. Topics Signal Process., vol. 12, no. 3, pp. 445-454, Jun 2018.
[8] X. Liu, Q. Zhang, W. Chen, H. Feng, L. Chen, F. M. Ghannouchi, and Z. Feng, "Beam-oriented digital predistortion for 5G massive MIMO hybrid beamforming transmitters," IEEE Trans. Microw. Theory Techn., vol. 66, no. 7, pp. 3419-3432, Jul 2018.

[9] L. Ding, G. T. Zhou, D. R. Morgan, Z. Ma, J. S. Kenney, J. Kim, and C. R. Giardina, "A robust digital baseband predistorter constructed using memory polynomials," IEEE Trans. Commun. Technol., vol. 52, no. 1, pp. $159-165$, Jan 2004

[10] A. S. Tehrani, H. Cao, S. Afsardoost, T. Eriksson, M. Isaksson, and C. Fager, "A comparative analysis of the complexity/accuracy tradeoff in power amplifier behavioral models," IEEE Trans. Microw. Theory Techn., vol. 58, no. 6, pp. 1510-1520, Jun 2010.

[11] R. N. Braithwaite, "A comparison of indirect learning and closed loop estimators used in digital predistortion of power amplifiers," in 2015 IEEE MTT-S International Microwave Symposium, May 2015, pp. 1-4.

[12] C. Mollen, E. G. Larsson, U. Gustavsson, T. Eriksson, and R. W. Heath, "Out-of-band radiation from large antenna arrays," IEEE Commun. Mag., vol. 56, no. 4, pp. 196-203, Apr 2018.

[13] N. D. Sidiropoulos, T. N. Davidson, and Z.-Q. Luo, "Transmit beamforming for physical-layer multicasting," IEEE Trans. Signal Process., vol. 54, no. 6, pp. 2239-2251, Jun 2006

[14] M. Grant and S. Boyd, "CVX: Matlab software for disciplined convex programming, version 2.1," http://cvxr.com/cvx, Mar. 2014.

[15] - "Graph implementations for nonsmooth convex programs," in Recent Advances in Learning and Control, ser. Lecture Notes in Control and Information Sciences, V. Blondel, S. Boyd, and H. Kimura, Eds. Springer-Verlag Limited, 2008, pp. 95-110, http://stanford.edu/ boyd/ graph_dcp.html. 